\title{
LIMIT CYCLES OF PIECEWISE SMOOTH DIFFERENTIAL EQUATIONS ON TWO DIMENSIONAL TORUS
}

\author{
JAUME LLIBRE ${ }^{1}$, RICARDO MIRANDA MARTINS ${ }^{2}$ AND DURVAL JOSÉ TONON ${ }^{3}$
}

\begin{abstract}
In this paper we study the limit cycles of some classes of piecewise smooth vector fields defined in the two dimensional torus. The piecewise smooth vector fields that we consider are composed by linear, Ricatti and perturbations of these two classes. For these kind of piecewise smooth vector fields we study their global dynamics, their upper bounds for the maximum number of limit cycles that they can exhibit, and the existence of non-trivial recurrences and of a continuum of periodic orbits. We also present a family of piecewise smooth vector fields that possesses a finite number of fold points, and that for any positive integer $k$ there are values of the parameters of this family for which the piecewise smooth vector field exhibit $k$ limit cycles,
\end{abstract}

\section{INTRODUCTION}

The theory of piecewise smooth vector fields (PSVF) has been studied intensively in these last years, mainly due to its strong relation with branches of applied sciences. These PSVF are in the boundary between mathematics, physics and engineering, for more details see for instance the two recent surveys [7] and [13], and the two books [4] and [12] on this subject, where also models of PSVF from control theory are considered. Roughly speaking the PSVF are formed by several smooth differential systems defined in different regions of the global domain of definition of the PSVF. The common frontier between the regions that separate the different smooth vector fields is called switching manifold (or discontinuity manifold).

Let $\mathbb{T}$ be the two dimensional torus. We decomposed $\mathbb{T}$ as the union of $\mathbb{T}^{+}$with $\mathbb{T}^{-}$, where $\mathbb{T}^{+}$denotes the closed upper half part of the torus $\mathbb{T}$ (homeomorphic to a closed annulus), and $\mathbb{T}^{-}$the closed bottom half part of this torus (also homeomorphic to a closed annulus). We denote by $\Sigma=\mathbb{T}^{+} \cap \mathbb{T}^{-}$a smooth curve, formed by two circles, which separates $\mathbb{T}$ into two connected components, each one homeomorphic to an open annulus. Let $X^{+}$and $X^{-}$be smooth vector fields on $\mathbb{T}^{+}$and $\mathbb{T}^{-}$, respectively. A precise definition of $\mathbb{T}, \mathbb{T}^{+}, \mathbb{T}^{-}$and $\Sigma$ is given at the beginning of Section 2 .

In this paper we consider piecewise smooth differential equations of the form

$$
\dot{\mathbf{x}}=\left\{\begin{array}{lll}
X^{+}(\mathbf{x}) & \text { if } & \mathbf{x} \in \mathbb{T}^{+}, \\
X^{-}(\mathbf{x}) & \text { if } & \mathbf{x} \in \mathbb{T}^{-} .
\end{array}\right.
$$

The dynamics over $\Sigma$ is defined following the Filippov's convention (see [5]). For simplicity a differential system (1) will be denoted by $\left(X^{+}, X^{-}\right)$, and referred as vector field (1).

2010 Mathematics Subject Classification. Primary 34A36, 34C07, 34C23, 34C60.

Key words and phrases. piecewise smooth differential equations, limit cycles, global dynamics in torus. 
The study of piecewise smooth dynamical systems defined on torus is not new, but as far as we know it has been restricted to the case of discrete dynamical systems. There are a large number of results for piecewise maps [1], [2], [3] and [14], but there is a lack of theoretical results for the case of piecewise dynamical systems where the flow is the solution of a piecewise differential system.

The research of the number and stability of limit cycles for some classes of vector fields is one of the most relevant problems of the qualitative theory of the dynamical systems. This kind of studies started with Poincaré in [11] and [10]. The main objective of this paper is to start this research first for the PSVF (1) when the smooth vector fields on $\mathbb{T}^{+}$and $\mathbb{T}^{-}$are either linear, or Ricatti, or some families of perturbations of them coming from the applications (see (3)), and after for a family of PSVF presenting a finite number of fold points (see (6)).

This paper is organized as follows. In Section 2 we formalize some basic concepts on the PSVF, as the first return map in this scenario and present some techniques that we shall use in the proof of the main results. In Section 3 the main results are presented, in Section 4 we prove these results, and in Section 5 we end this paper presenting some numerical examples of PSVF with the maximum number of limit cycles that they can exhibit.

\section{BASIC THEORY}

2.1. Filippov's convention. In this work the two dimensional torus $\mathbb{T}$ that we consider is defined by the following equivalence relation in the square $Q=[0,1] \times[0,1] \subset \mathbb{R}^{2}$ :

$$
(x, y) \sim(z, w) \Leftrightarrow(x-z, y-w) \in \mathbb{Z} \times \mathbb{Z} .
$$

Consider $\Sigma_{1}=\{(x, y) \in Q: y=0\}$ and $\Sigma_{2}=\{(x, y) \in Q: y=1 / 2\}$. We denote by $h_{1}(x, y)=y$ and $h_{2}(x, y)=y-1 / 2$, in this way we can write $\Sigma_{1}=h_{1}^{-1}(0)$ and $\Sigma_{2}=h_{2}^{-1}(0)$. Clearly the switching manifold $\Sigma=\Sigma_{1} \cup \Sigma_{2}$ is the common boundary between the two regions $\mathbb{T}^{-}=\{(x, y) \in \mathbb{T} ; 0 \leq y \leq 1 / 2\}$ and $\mathbb{T}^{+}=\{(x, y) \in \mathbb{T} ; 1 / 2 \leq y \leq 1\}$.

Designate by $\mathfrak{X}^{r}$ the space of $C^{r}$-vector fields on $\mathbb{T}$ endowed with the $C^{r}$-topology with $r=\infty$ or $r \geq 1$ large enough for our purposes. Call $\Omega^{r}$ the space of PSVF $X: \mathbb{T} \rightarrow \mathbb{T}$ such that

$$
X(x, y)=\left\{\begin{array}{lll}
X^{+}(x, y) & \text { for } & (x, y) \in \mathbb{T}^{+} \\
X^{-}(x, y) & \text { for } & (x, y) \in \mathbb{T}^{-}
\end{array}\right.
$$

where $X^{+}=\left(X_{1}^{+}, X_{2}^{+}\right)$and $X^{-}=\left(X_{1}^{-}, X_{2}^{-}\right)$are in $\mathfrak{X}^{r}$. Let $h \in\left\{h_{1}, h_{2}\right\}$. We denote by $X^{ \pm} h(p)=\left\langle X^{ \pm}(p), \nabla h(p)\right\rangle$ and $\left(X^{ \pm}\right)^{n} h(p)=\left\langle X^{ \pm}(p), \nabla\left(X^{ \pm}\right)^{n-1} h(p)\right\rangle$ the Lie's derivatives, where $\langle\cdot, \cdot\rangle$ denote the Euclidean inner product. We may consider $\Omega^{r}=\mathfrak{X}^{r} \times \mathfrak{X}^{r}$ endowed with the product topology and denote any element in $\Omega^{r}$ by $X=\left(X^{+}, X^{-}\right)$, which we will accept to be multivalued in points of $\Sigma$. In this context the basic results on the PSVF were stated by Filippov in [5]. Related theories can be found in $[4,9,13]$ and references therein.

On $\Sigma$ we generically distinguish three regions: the crossing region $\Sigma^{c}=\left\{p \in \Sigma: X_{2}^{+}(p)\right.$ $\left.X_{2}^{-}(p)>0\right\}$, the stable sliding region $\Sigma^{s}=\left\{p \in \Sigma: X_{2}^{+}(p)<0, X_{2}^{-}(p)>0\right\}$, and the unstable sliding region $\Sigma^{u}=\left\{p \in \Sigma: X_{2}^{+}(p)>0, X_{2}^{-}(p)<0\right\}$.

Following the Filippov's convention if $q \in \Sigma^{s}$ the sliding vector field associated to $X \in \Omega^{r}$ is the vector field $\widehat{X}^{s}$ tangent to $\Sigma^{s}$, expressed in coordinates as

$$
\widehat{X}^{s}(q)=\frac{1}{\left(X_{2}^{-}-X_{2}^{+}\right)(q)}\left(\left(X_{1}^{+}-X_{1}^{-}\right)(q), 0\right),
$$


which, after a time rescaling, is topologically equivalent to the normalized sliding vector field

$$
X^{s}(q)=\left(X_{1}^{+}-X_{1}^{-}\right)(q) .
$$

A point $q \in \Sigma$ such that $X^{s}(q)=0$ is called a pseudo equilibrium of $X$, and a point $p \in \Sigma$ such that $X^{+} h(p) X^{-} h(p)=0$ is called a tangential singularity of $X$ (i.e. the trajectory through $p$ is tangent to $\Sigma)$. We say that a point $q \in \Sigma$ is a regular point if $q \in \Sigma^{c}$ or $q \in \Sigma^{s}$ and $X^{s}(q) \neq 0$.

A tangential singularity $q \in \Sigma$ of $X^{+}$is a fold point of $X^{+}$if $X^{+} h(q)=0$ but $\left(X^{+}\right)^{2} h(q) \neq 0$, visible tangency if $\left(X^{+}\right)^{2} h(q)>0$ and invisible tangency if $\left(X^{+}\right)^{2} h(q)<0$.

The flow $\phi_{X}$ of $X \in \Omega^{r}$ is obtained by the concatenation of flows of $X^{+}, X^{-}$and $X^{s}$, denoted by $\phi_{X^{+}}, \phi_{X^{-}}$and $\phi_{X^{s}}$, respectively.

Let be $X=\left(X^{+}, X^{-}\right) \in \Omega^{r}$, we say that $p \in \Sigma$ is a fold-regular point of $X$ if $p$ is a fold point of $X^{+}$and $X^{-}(p)$ is transversal to $\Sigma$ at $p$.

2.2. Extended Chebyshev systems. Let $I$ be a proper real interval. A ordered set of functions $\mathcal{F}=\left\{g_{j}: I \rightarrow \mathbb{R}\right.$ for $\left.j=0,1, \ldots, k\right\}$ is an extended Chebyshev system on $I$ if and only if every nontrivial linear combination of functions of $\mathcal{F}$ has at most $k$ zeros taking into account their multiplicities. $\mathcal{F}$ is an extended complete Chebyshev system on $I$ if and only if for any $s$, $0 \leq s \leq k$, we get that $\left(g_{0}, g_{1}, \ldots, g_{s}\right)$ is an extended system. For details and proofs see [6].

It is necessary and sufficient for proving that $\mathcal{F}$ is an extended Chebyshev system on $I$ that $W\left(g_{0}, g_{1}, \ldots, g_{s}\right)(t) \neq 0$ on $I$ for $0 \leq s \leq k$, where $W_{s}(t)=W\left(g_{0}, g_{1}, \ldots, g_{s}\right)(t)$ is the Wronskian of the functions $\left(g_{0}, g_{1}, \ldots, g_{s}\right)$ with respect to $t$.

In [8] the authors proved that for a family of $n+1$ linearly independent analytical functions where at least one of that possess constant sign in its domain, there exists a linear combination of these functions having at least $n$ simple zeros. Precisely, they proved the following result:

Theorem B Let $\mathcal{F}=\left\{g_{0}, g_{1}, \ldots, g_{n}\right\}$ be an ordered set of real $C^{\infty}$ functions on $(a, b)$ for which there exists $\xi \in(a, b)$ with $W\left(g_{0}, g_{1}, \ldots, g_{n-1}\right)(\xi)=W_{n-1}(\xi) \neq 0$. Then the following statements hold.

(a) If $W_{n}(\xi) \neq 0$ then for each configuration of $m \leq n$ zeros, taking into account their multiplicity, there exists a linear combination of the functions of $\mathcal{F}$ having this configuration of zeros.

(b) If $W_{n}(\xi)=0$ and $W_{n}^{\prime}(\xi) \neq 0$ then for each configuration of $m \leq n+1$ zeros, taking into account their multiplicity, there exists a linear combination of the functions of $\mathcal{F}$ having this configuration of zeros.

\section{MAIN RESUlts FOR PSVF IN THE TWO DIMENSIONAL TORUS}

One of the main objectives of this paper is to study the linear and Ricatti vector fields in $\mathbb{T}$, that we denote by

$$
\begin{aligned}
& X_{L}^{\omega}(x, y)=\left(a^{\omega} y+b^{\omega}, c^{\omega} y+d^{\omega}\right), \\
& X_{R}^{\omega}(x, y)=\left(1, e^{\omega}+f^{\omega} y+g^{\omega} y^{2}\right),
\end{aligned}
$$

respectively, where $a^{\omega}, b^{\omega}, c^{\omega}, d^{\omega}, e^{\omega}, f^{\omega}, g^{\omega} \in \mathbb{R}$ and either $\omega=+$ or $\omega=-$, if the vector field is defined either in $\mathbb{T}^{+}$or in $\mathbb{T}^{-}$. The special case of $X_{L}^{\omega}$ where $a^{\omega}=c^{\omega}=0$ in $X_{L}^{\omega}$ will be denoted by $X_{C}^{\omega}$ (constant vector field). 
In the following we shall perturb these PSVF considering the functions defined in $\mathbb{T}$ :

$$
F_{1}(x, y)=\left(-x+x^{2}, 0\right), \quad F_{2}(x, y)=\left(\eta_{1} y+\eta_{2} y^{2}, 0\right), \quad F_{3}(x, y)=(\cos (2 \pi x), 0),
$$

where $\eta_{1}, \eta_{2} \in \mathbb{R}$ and are small. We denote by $X_{L L}$ the PSVF composed by two linear vector fields in each half torus, by $X_{L R}$ the PSVF composed by a linear vector field in $\mathbb{T}^{-}$and Ricatti vector field on $\mathbb{T}^{+}$, and by $X_{R R}$ the PSVF composed by the Ricatti vector fields in each half torus. Considering the PSVFs $X_{L L}, X_{L R}$ and $X_{R R}$ we perform the following perturbations:

$$
\begin{aligned}
X_{R R 3+} & =X_{R R}+\varepsilon\left(F_{3}, \overrightarrow{0}\right), \quad X_{L R 1-}=X_{L R}+\varepsilon\left(\overrightarrow{0}, F_{1}\right), \quad X_{L R 2+}=X_{L R}+\left(F_{2}, \overrightarrow{0}\right), \\
X_{L R 2-} & =X_{L R}+\left(\overrightarrow{0}, F_{2}\right), \quad X_{L R 3-}=X_{L R}+\varepsilon\left(\overrightarrow{0}, F_{3}\right),
\end{aligned}
$$

where $\overrightarrow{0}$ denotes the null vector field $(0,0)$ in $\mathbb{T}$.

Remark 1. We only consider perturbations of $X_{L L}$ and $X_{R R}$ in $\mathbb{T}^{+}$, because due to the symmetry of the problem we should obtain the same results if we consider perturbations in $\mathbb{T}^{-}$.

For each of the families presented in (4) we consider the following subfamilies

$$
\begin{aligned}
& \Omega_{L^{\omega}}^{1}=\left\{X_{L^{\omega}} ; c^{\omega}>0, d^{\omega}>-\frac{c^{\omega}}{2}\right\}, \\
& \Omega_{L^{\omega}}^{2}=\left\{X_{L^{\omega}} ; c^{\omega}<0,-1<\frac{c^{\omega}}{\left(c^{\omega}+2 d^{\omega}\right)}<0\right\}, \\
& \Omega_{L^{\omega}}^{3}=\left\{X_{L^{\omega}} ; c^{\omega}<0,-1<\frac{c^{\omega}}{d^{\omega}}<0\right\}, \\
& \Omega_{L^{\omega}}^{4}=\left\{X_{L^{\omega}} ; c^{\omega}>0, d^{\omega}>0\right\} \\
& \Omega_{L^{\omega}}^{5}=\left\{X_{L^{\omega}} ; a^{\omega}>0, b^{\omega}>0\right\}, \\
& \Omega_{L^{\omega}}^{6}=\left\{X_{L^{\omega}} ; a^{\omega}<0,-1<\frac{a^{\omega}}{\left(2 b^{\omega}\right)}<0\right\}, \\
& \Omega_{R^{\omega}}^{1}=\left\{X_{R^{\omega}} ; f^{\omega}>0, e^{\omega} g^{\omega}>\left(\frac{f^{\omega}}{2}\right)^{2}, \tan ^{-1}\left(\theta_{1}^{\omega}\right)>\tan ^{-1}\left(\theta_{2}^{\omega}\right)\right\}, \\
& \Omega_{R^{\omega}}^{2}=\left\{X_{R^{\omega}} ; f^{\omega}>0, e^{\omega} g^{\omega}>\left(\frac{f^{\omega}}{2}\right)^{2}, \tan ^{-1}\left(\theta_{2}^{\omega}\right)>\sec ^{-1}\left(\theta_{3}^{\omega}\right)\right\}, \\
& \Omega_{R^{\omega}}^{3}=\left\{X_{R^{\omega}} ; f^{\omega}>0, e^{\omega} g^{\omega}>\left(\frac{f^{\omega}}{2}\right)^{2}, \tan ^{-1}\left(\theta_{1}^{\omega}\right)>\sec ^{-1}\left(\theta_{3}^{\omega}\right)\right\},
\end{aligned}
$$

where $\theta_{1}^{\omega}=\frac{f^{\omega}+2 g^{\omega}}{\sqrt{4 e^{\omega} g^{\omega}-\left(f^{\omega}\right)^{2}}}, \theta_{2}^{\omega}=\frac{f^{\omega}+g^{\omega}}{\sqrt{4 e^{\omega} g^{\omega}-\left(f^{\omega}\right)^{2}}}$ and $\theta_{3}^{\omega}=\frac{2 \sqrt{e^{\omega} g^{\omega}}}{\sqrt{4 e^{\omega} g^{\omega}-\left(f^{\omega}\right)^{2}}}$. Prior to present the theorem we define the following real numbers

$$
\begin{aligned}
\Delta_{L L} & =\frac{1}{2\left(c^{-} c^{+}\right)^{2}}\left(c^{-}\left(c^{+}\left(a^{-} c^{+}+a^{+} c^{-}\right)+2 c^{-} \log \left(\frac{c^{+}}{c^{+}+2 d^{+}}+1\right)\left(b^{+} c^{+}-a^{+} d^{+}\right)\right)\right. \\
& \left.+2\left(c^{+}\right)^{2} \log \left(\frac{c^{-}}{2 d^{-}}+1\right)\left(b^{-} c^{-}-a^{-} d^{-}\right)\right)
\end{aligned}
$$




$$
\begin{aligned}
& \Delta_{L R}=\frac{1}{2\left(c^{-}\right)^{2}}\left(a^{-} c^{-}+\left(4\left(c^{-}\right)^{2}\left(-\tan ^{-1}\left(\theta_{2}^{+}\right)+\tan ^{-1}\left(\theta_{1}^{+}\right)\right) / \sqrt{-\left(f^{+}\right)^{2}+4 e^{+} g^{+}}\right.\right. \\
& \left.+2 b^{-} c^{-} \log \left(1+c^{-} /\left(2 d^{-}\right)\right)-2 a^{-} d^{-} \log \left(1+c^{-} /\left(2 d^{-}\right)\right)\right), \\
& \Delta_{R R}=\frac{\sec ^{-1}\left(\theta_{3}^{-}\right)-\tan ^{-1}\left(\theta_{2}^{-}\right)}{\sqrt{4 e^{-} g^{-}-\left(f^{-}\right)^{2}}}+\frac{\tan ^{-1}\left(\theta_{2}^{+}\right)-\tan ^{-1}\left(\theta_{1}^{+}\right)}{\sqrt{4 e^{+} g^{+}-\left(f^{+}\right)^{2}}}, \\
& \Delta_{L L 2+}=\frac{1}{8\left(c^{+}\right)^{3}}\left(8 \log \left(\frac{c^{+}}{c^{+}+2 d^{+}}+1\right)\left(d^{+}\left(d^{+} \eta_{2}-c^{+}\left(a^{+}+\eta_{1}\right)\right)+b^{+}\left(c^{+}\right)^{2}\right)+\right. \\
& \left.c^{+}\left(4 c^{+}\left(a^{+}+c^{+}+\eta_{1}\right)+\eta_{2}\left(3 c^{+}-4 d^{+}\right)\right)\right) \text {, } \\
& \Delta_{R R 2+}=\frac{1}{2\left(g^{+}\right)^{2} \sqrt{4 e^{-} g^{-}-\left(f^{-}\right)^{2}} \sqrt{4 e^{+} g^{+}-\left(f^{+}\right)^{2}}}\left(\sqrt{4 e^{-} g^{-}-\left(f^{-}\right)^{2}}\right. \\
& \left(\tan ^{-1}\left(\theta_{2}^{+}\right)\left(4 e^{+} \eta_{2} g^{+}-2\left(f^{+}\right)^{2} \eta_{2}+2 f^{+} \eta_{1} g^{+}-4\left(g^{+}\right)^{2}\right)+\right. \\
& 2 \tan ^{-1}\left(\theta_{1}^{+}\right)\left(-g^{+}\left(2 e^{+} \eta_{2}+f^{+} \eta_{1}\right)+\left(f^{+}\right)^{2} \eta_{2}+2\left(g^{+}\right)^{2}\right)+ \\
& \sqrt{4 e^{+} g^{+}-\left(f^{+}\right)^{2}}\left(\log \left(\frac{g^{+}\left(e^{+}+f^{+}+g^{+}\right)}{4 e^{+} g^{+}-\left(f^{+}\right)^{2}}\right)\left(\eta_{1} g^{+}-f^{+} \eta_{2}\right)+\right. \\
& \left.\left.\log \left(1+\left(\theta_{2}^{+}\right)^{2}\right)\left(f^{+} \eta_{2}-\eta_{1} g^{+}\right)-f^{+} \eta_{2} \log (4)+\eta_{1} g^{+} \log (4)+\eta_{2} g^{+}\right)\right)+ \\
& \left.4\left(g^{+}\right)^{2} \sqrt{4 e^{+} g^{+}-\left(f^{+}\right)^{2}}\left(\tan ^{-1}\left(\theta_{2}^{-}\right)-\sec ^{-1}\left(\theta_{3}^{-}\right)\right)\right) \text {, } \\
& \Delta_{L R 2-}=\frac{1}{2\left(c^{+}\right)^{2}\left(g^{-}\right)^{2} \sqrt{4 e^{-} g^{-}-\left(f^{-}\right)^{2}}}\left(\sqrt { 4 e ^ { - } g ^ { - } - ( f ^ { - } ) ^ { 2 } } \left(2\left(g^{-}\right)^{2} \log \left(\frac{c^{+}}{c^{+}+2 d^{+}}+1\right)\right.\right. \\
& \left(b^{+} c^{+}-a^{+} d^{+}\right)+c^{+}\left(a^{+}\left(g^{-}\right)^{2}+2 c^{+} \eta_{1} g^{-} \log \left(\frac{\sqrt{4 e^{-} g^{-}-\left(f^{-}\right)^{2}}}{\sqrt{e^{-}} \sqrt{g^{-}}}\right)-\right. \\
& 2 c^{+} \eta_{1} g^{-} \log \left(\frac{1}{\sqrt{1+\left(\theta_{2}^{-}\right)^{2}}}\right)-c^{+} \sqrt{e^{-}} \eta_{2} \sqrt{g^{-}} \sqrt{\frac{(f-)^{2}}{e^{-} g^{-}}}- \\
& 2 c^{+} f^{-} \eta_{2} \log \left(\frac{\sqrt{4 e^{-} g^{-}-\left(f^{-}\right)^{2}}}{2 \sqrt{e^{-} g^{-}}}\right)+2 c^{+} f^{-} \eta_{2} \log \left(\frac{1}{\sqrt{1+\left(\theta_{2}^{+}\right)^{2}}}\right)+ \\
& \left.\left.c^{+} f^{-} \eta_{2}-c^{+} \eta_{1} g^{-} \log (4)+c^{+} \eta_{2} g^{-}\right)\right)+\left(2 ( c ^ { + } ) ^ { 2 } \left(-g^{-}\left(2 e^{-} \eta_{2}+f^{-} \eta_{1}\right)+\right.\right. \\
& \left.\left.\left.\left(f^{-}\right)^{2} \eta_{2}+2\left(g^{-}\right)^{2}\right)\right)\left(\tan ^{-1}\left(\theta_{2}^{-}\right)-\sec ^{-1}\left(\theta_{3}^{-}\right)\right)\right) \text {, } \\
& \Delta_{L R 2+}=\frac{1}{8\left(c^{+}\right)^{3}\left(\left(f^{-}\right)^{2}-4 e^{-} g^{-}\right)}\left(( ( f ^ { - } ) ^ { 2 } - 4 e ^ { - } g ^ { - } ) \left(8 \log \left(\frac{c^{+}}{c^{+}+2 d^{+}}+1\right)\right.\right. \\
& \left.\left.\left(c^{+}\left(b^{+} c^{+}-d^{+}\left(a^{+}+\eta_{1}\right)\right)+\left(d^{+}\right)^{2} \eta_{2}\right)+c^{+}\left(4 c^{+} a^{+}+\eta_{1}\right)+\eta_{2}\left(3 c^{+}-4 d^{+}\right)\right)\right)+ \\
& \left.16\left(c^{+}\right)^{3} \sqrt{4 e^{-} g^{-}-\left(f^{-}\right)^{2}}\left(\sec ^{-1}\left(\theta_{3}^{-}\right)-\tan ^{-1}\left(\theta_{2}^{-}\right)\right)\right) .
\end{aligned}
$$

In Theorem 2 we prove that these subfamilies correspond the piecewise smooth vector fields where the first return map $P: \Sigma_{1} \rightarrow \Sigma_{1}$ is defined.

Theorem 2. Consider the PSVFs defined in (5).

(a) If $\Delta_{L L} \in \mathbb{Q}$ then $X_{L L}$ has a continuum of periodic orbits, and if $\Delta_{L L} \notin \mathbb{Q}$ then all trajectories of $X_{L L}$ are dense.

(b) If $\Delta_{L R} \in \mathbb{Q}$ then $X_{L R}$ has a continuum of periodic orbits, and if $\Delta_{L R} \notin \mathbb{Q}$ then all trajectories of $X_{L R}$ are dense. 
(c) If $\Delta_{R R} \in \mathbb{Q}$ then $X_{R R}$ has a continuum of periodic orbits, and if $\Delta_{R R} \notin \mathbb{Q}$ then all trajectories of $X_{R R}$ are dense.

Considering the perturbations $F_{i}^{\omega}$ we have

(d) If $\Delta_{L L 2+} \in \mathbb{Q}$ then $X_{L L 2+}$ has a continuum of periodic orbits, and if $\Delta_{L L 2+} \notin \mathbb{Q}$ then all trajectories of $X_{L L 2+}$ are dense.

(e) If $\varepsilon>0$ then the maximum number of limit cycles for $X_{R R 1+}$ is two, and this upper bound is reached.

( $f$ ) If $\Delta_{R R 2+} \in \mathbb{Q}$ then $X_{R R 2+}$ has a continuum of periodic orbits, and if $\Delta_{R R 2+} \notin \mathbb{Q}$ then all trajectories of $X_{R R 2+}$ are dense.

(g) The maximum number of limit cycles of $X_{R R 3+}$ is two, and this upper bound is reached.

(h) The maximum number of limit cycles of $X_{L R 1-}$ is two, and this upper bound is reached if $X_{L R 1-} \in\left[\Omega_{L^{+}}^{1} \cup \Omega_{L^{+}}^{2}\right] \cap \Omega_{R^{-}}^{2}, \varepsilon<0, a^{+} c^{+}>0$ and $b^{+} c^{+}>a^{+} d^{+}$.

(i) If $\Delta_{L R 2-} \in \mathbb{Q}$ then $X_{L R 2-}$ has a continuum of periodic orbits, and if $\Delta_{L R 2-} \notin \mathbb{Q}$ then all trajectories of $X_{L R 2-}$ are dense.

(j) The maximum number of limit cycles of $X_{L R 3-}$ is two, and this upper bound is reached.

(l) If $\Delta_{L R 2+} \in \mathbb{Q}$ then $X_{L R 2+}$ has a continuum of periodic orbits, and if $\Delta_{L R 2+} \notin \mathbb{Q}$ then all trajectories of $X_{L R 2+}$ are dense.

In what follows we consider a PSVF $X_{C k}=\left(X_{C}, X_{k}\right)$ in $\mathbb{T}$ having a finite number of foldregular points in $\Sigma$, where

$$
X_{k}(x, y)=(\alpha, \beta \cos (2 k \pi x)),
$$

is defined in $\mathbb{T}^{-}$and $X_{C}(x, y)=\left(b^{+}, d^{+}\right)$is defined in $\mathbb{T}^{+}$, with $b^{+}, d^{+} \in \mathbb{R}, k$ is a positive integer and $\alpha, \beta \in \mathbb{R}$. For this PSVF there exists a choice of the parameters of $X_{C k}$ such that $X_{C k}$ exhibits a finite number of limit cycles depending on $k$. More precisely we have the following result.

Theorem 3. The PSVF $X_{C k}$ has at most $k$ limit cycles, and this upper bound is reached for every $k \geq 1$.

Remark 4. Note that the vector field in the family $X_{C k}$ can have no limit cycles. In such case there are sliding regions over the switching manifold and $X_{C k}$ may present a chaotic behavior, see for instance [15].

\section{Proof of main Results}

4.1. Preliminary results. Before to prove the main results of this paper we need some auxiliary results. The next lemma provides the expression of the first return map for the PSVFs $X_{L L}, X_{R R}, X_{L R}$ and their perturbations.

Lemma 5. Consider the PSVFs defined in (5) and the functions defined in (3). 
(a) If $X_{L L} \in\left[\Omega_{L^{+}}^{1} \cup \Omega_{L^{+}}^{2}\right] \cap\left[\Omega_{L^{-}}^{1} \cup \Omega_{L^{-}}^{2}\right]$ then the first return map $P_{L L}: \Sigma_{1} \rightarrow \Sigma_{1}$ is well defined and is given by $P_{L L}\left(x_{0}\right)=x_{0}+\Delta_{L L}$.

(b) If $X_{L R} \in\left[\Omega_{L^{-}}^{1} \cup \Omega_{L^{+}}^{2}\right] \cap \Omega_{R^{+}}^{1}$ then the first return map $P_{L R}: \Sigma_{1} \rightarrow \Sigma_{1}$ is well defined and is given by $P_{L R}\left(x_{0}\right)=x_{0}+\Delta_{L R}$.

(c) If $X_{R R} \in\left[\Omega_{R^{+}}^{1} \cap \Omega_{R^{-}}^{2}\right]$ then the first return map $P_{R R}: \Sigma_{1} \rightarrow \Sigma_{1}$ is well defined and is given by $P_{R R}\left(x_{0}\right)=x_{0}+\Delta_{R R}$.

(d) If $X_{L L 2+} \in\left[\Omega_{L^{-}}^{5} \cup \Omega_{L^{-}}^{6}\right] \cap\left[\Omega_{L^{+}}^{1} \cup \Omega_{L^{+}}^{2}\right]$ then the first return map $P_{L L 2+}: \Sigma_{1} \rightarrow \Sigma_{1}$ is well defined and is given by $P_{L L 2+}\left(x_{0}\right)=x_{0}+\Delta_{L L 2+}$

(e) If $X_{R R 1+} \in\left[\Omega_{R^{-}}^{2} \cap \Omega_{R^{+}}^{3}\right]$ and $\varepsilon$ is a small positive number then the first return map $P_{R R 1+}: \Sigma_{1} \rightarrow \Sigma_{1}$ is well defined and is given by

$$
\begin{aligned}
P_{R R 1+}\left(x_{0}\right)= & \frac{1}{2 \sqrt{-(\varepsilon-4) \varepsilon}}\left(\sqrt{(4-\varepsilon) \varepsilon}+(4-\varepsilon) \tan \left(\frac{\sqrt{-(\varepsilon-4) \varepsilon}\left(\tan ^{-1}\left(\theta_{1}^{+}\right)-\sec ^{-1}\left(\theta_{3}^{+}\right)\right)}{\sqrt{4 e^{+} g^{+}-\left(f^{+}\right)^{2}}}-\right.\right. \\
& \tan ^{-1}\left(\frac { 1 } { \sqrt { 4 - \varepsilon } ( ( ( f ^ { - } ) ^ { 2 } - 4 e ^ { - } g ^ { - } ) } \sqrt { \varepsilon } \left(\left(2 x_{0}-1\right)\left(\left(-\left(f^{-}\right)^{2}+4 e^{-} g^{-}\right)\right)+\right.\right. \\
& \left.\left.\left.\left.\left.4 \sqrt{4 e^{-} g^{-}-\left(f^{-}\right)^{2}} \tan ^{-1}\left(\theta_{2}^{-}\right)-4 \sqrt{4 e^{-} g^{-}-\left(f^{-}\right)^{2}} \sec ^{-1}\left(\theta_{3}^{-}\right)\right)\right)\right)\right)\right) .
\end{aligned}
$$

(f) If $X_{R R 2+} \in\left[\Omega_{R^{-}}^{1} \cap \Omega_{R^{+}}^{2}\right]$ then the first return map $P_{R R 2+}: \Sigma_{1} \rightarrow \Sigma_{1}$ is well defined and is given by $P_{R R 2+}\left(x_{0}\right)=x_{0}+\Delta_{R R 2+}$

(g) If $X_{R R 3+} \in\left[\Omega_{R^{-}}^{2} \cap \Omega_{R^{+}}^{1}\right]$ and $\varepsilon$ is a small positive number then the first return map $P_{R R 3+}: \Sigma_{1} \rightarrow \Sigma_{1}$ is well defined and is given by

$$
\begin{aligned}
P_{R R 3+}\left(x_{0}\right)= & -\frac{1}{\pi} \tan ^{-1}\left(\frac{\sqrt{\varepsilon+1}}{\sqrt{1-\varepsilon}}\left(\tan \left(\frac{2 \pi \sqrt{1-\varepsilon^{2}}\left(\tan ^{-1}\left(\theta_{2}^{+}\right)-\tan ^{-1}\left(\theta_{1}^{+}\right)\right)}{\sqrt{4 e^{+} g^{+}-\left(f^{+}\right)^{2}}}\right)\right)-\right. \\
& \tan ^{-1}\left(\frac { ( \varepsilon - 1 ) } { \sqrt { 1 - \varepsilon ^ { 2 } } } \operatorname { t a n } \left(\frac { \pi } { ( f ^ { - } ) ^ { 2 } - 4 e ^ { - } g ^ { - } } \left(-\left(f^{-}\right)^{2}+2 \sqrt{4 e^{-} g^{-}-\left(f^{-}\right)^{2}}\right.\right.\right. \\
& \left.\left.\left.\left.\tan ^{-1}\left(\theta_{2}^{+}\right)-2 \sqrt{4 e^{-} g^{-}-\left(f^{-}\right)^{2}} \sec ^{-1}\left(\theta_{3}^{-}\right)+4 e^{-} g^{-} x_{0}-\left(f^{-}\right)^{2} x_{0}\right)\right)\right)\right)
\end{aligned}
$$

(h) If $X_{L R 1_{-}} \in\left[\Omega_{L^{+}}^{1} \cup \Omega_{L^{+}}^{2}\right] \cap \Omega_{R^{-}}^{2}$ and $\varepsilon$ is a small negative number then the first return map $P_{L R 1-}: \Sigma_{1} \rightarrow \Sigma_{1}$ is well defined and is given by

$$
\begin{aligned}
P_{L R 1-}\left(x_{0}\right) & =\frac{1}{2}\left(\frac{2 \log \left(\frac{c^{+}}{c^{+}+2 d^{+}}+1\right)\left(b^{+} c^{+}-a^{+} d^{+}\right)+c^{+}\left(a^{+}+c^{+}\right)}{\left(c^{+}\right)^{2}}+\right. \\
& \left.\frac{\tanh \left(\frac{\sqrt{4-\varepsilon} \sqrt{-\varepsilon}\left(\tan ^{-1}\left(\theta_{2}^{+}\right)-\sec ^{-1}\left(\theta_{3}^{-}\right)\right)}{\sqrt{4 e^{-} g^{-}-\left(f^{-}\right)^{2}}}+\tanh ^{-1}\left(\left(2 x_{0}-1\right) \sqrt{\frac{\varepsilon}{\varepsilon-4}}\right)\right)}{\sqrt{\frac{\varepsilon}{\varepsilon-4}}}\right) .
\end{aligned}
$$

(i) If $X_{L R 2-} \in\left[\Omega_{L^{+}}^{1} \cup \Omega_{L^{+}}^{2}\right] \cap \Omega_{R^{-}}^{2}$ then the first return map $P_{L R 2-}: \Sigma_{1} \rightarrow \Sigma_{1}$ is well defined and is given by $P_{L R 2-}\left(x_{0}\right)=x_{0}+\Delta_{L R 2-}$. 
(j) If $X_{L R 3_{-}} \in\left[\Omega_{L^{+}}^{1} \cup \Omega_{L^{+}}^{2}\right] \cap \Omega_{R^{-}}^{2}$ and $\varepsilon$ is a small positive number then the first return map $P_{L R 3-}: \Sigma_{1} \rightarrow \Sigma_{1}$ is well defined and is given by

$$
\begin{aligned}
P_{L R 3-}\left(x_{0}\right) & =\frac{1}{2 \pi\left(c^{+}\right)^{2}}\left(\pi\left(2 \log \left(\frac{c^{+}}{c^{+}+2 d^{+}}+1\right)\left(b^{+} c^{+}-a^{+} d^{+}\right)+a^{+} c^{+}\right)+\right. \\
& 2\left(c^{+}\right)^{2} \tan ^{-1}\left(\frac { \sqrt { \varepsilon + 1 } } { \sqrt { 1 - \varepsilon } } \operatorname { t a n } \left(\frac{2 \pi \sqrt{1-\varepsilon^{2}}\left(\tan ^{-1}\left(\theta_{2}^{-}\right)-\sec ^{-1}\left(\theta_{3}^{-}\right)\right)}{\sqrt{4 e^{-} g^{-}-\left(f^{-}\right)^{2}}}-\right.\right. \\
& \left.\left.\left.\tan ^{-1}\left(\frac{(\varepsilon-1) \tan \left(\pi x_{0}\right)}{\sqrt{1-\varepsilon^{2}}}\right)\right)\right)\right) .
\end{aligned}
$$

(l) If $X_{L R 2+} \in\left[\Omega_{L^{+}}^{1} \cup \Omega_{L^{+}}^{2}\right] \cap \Omega_{R^{-}}^{2}$ then the first return map $P_{L R 2+}: \Sigma_{1} \rightarrow \Sigma_{1}$ is well defined and is given by $P_{L R 2+}\left(x_{0}\right)=x_{0}+\Delta_{L R 2+}$.

Proof. The flow $\phi_{X}(t)$ where $X$ is one of the vector fields $X_{L \omega}, X_{R \omega}, X_{L 2 \omega}, X_{R 1 \omega}, X_{R 2 \omega}, X_{R 3 \omega}$ passing through the point $p=\left(x_{0}, y_{0}\right)$ when $t=0$ is given by

$$
\begin{aligned}
& \phi_{X_{L \omega}}(t)=\left(\frac { 1 } { ( c ^ { \omega } ) ^ { 2 } } \left(-a^{\omega} c^{\omega} d^{\omega} t+a^{\omega} d^{\omega} e^{c^{\omega} t}+a^{\omega} c^{\omega} y_{0} e^{c^{\omega} t}-a^{\omega} c^{\omega} y_{0}-a^{\omega} d^{\omega}+\right.\right. \\
& \left.\left.b^{\omega}\left(c^{\omega}\right)^{2} t+\left(c^{\omega}\right)^{2} x_{0}\right), \frac{d^{\omega} e^{c^{\omega} t}+c^{\omega} y_{0} e^{c^{\omega} t}-d^{\omega}}{c^{\omega}}\right), \\
& \phi_{X_{R}}(t)=\left(t+x_{0}, \frac{1}{2 g^{\omega}}\left(\sqrt { 4 e ^ { \omega } g ^ { \omega } - ( f ^ { \omega } ) ^ { 2 } } \operatorname { t a n } \left(\frac{1}{2} t \sqrt{4 e^{\omega} g^{\omega}-\left(f^{\omega}\right)^{2}}+\right.\right.\right. \\
& \left.\left.\left.\tan ^{-1}\left(\frac{f^{\omega}+2 g^{\omega} y_{0}}{\sqrt{4 e^{\omega} g^{\omega}-\left(f^{\omega}\right)^{2}}}\right)\right)-f^{\omega}\right)\right) \\
& \phi_{X_{L 2}}(t)=\left(\frac{e^{c^{\omega} t}\left(c^{\omega} y_{0}+d^{\omega}\right)-d^{\omega}}{c^{\omega}}, \frac{1}{2\left(c^{\omega}\right)^{3}}\left(-2 a^{\omega}\left(c^{\omega}\right)^{2} d^{\omega} t-2 a^{\omega}\left(c^{\omega}\right)^{2} y_{0}+\right.\right. \\
& 2 e^{c^{\omega} t}\left(c^{\omega} y_{0}+d^{\omega}\right)\left(c^{\omega}\left(a^{\omega}+\eta_{1}\right)-2 d^{\omega} \eta_{2}\right)-2 a^{\omega} c^{\omega} d^{\omega}+2 b^{\omega}\left(c^{\omega}\right)^{3} t+ \\
& 2\left(c^{\omega}\right)^{3} x_{0}-2\left(c^{\omega}\right)^{2} d^{\omega} \eta_{1} t-\left(c^{\omega}\right)^{2} \eta_{2} y_{0}^{2}-2\left(c^{\omega}\right)^{2} \eta_{1} y_{0}+2 c^{\omega}\left(d^{\omega}\right)^{2} \eta_{2} t- \\
& \left.2 c^{\omega} d^{\omega} \eta_{1}+\eta_{2} e^{2 c^{\omega} t}\left(c^{\omega} y_{0}+\left(d^{\omega}\right)^{2}+2 c^{\omega} d^{\omega} \eta_{2} y_{0}+3\left(d^{\omega}\right)^{2} \eta_{2}\right)\right), \\
& \phi_{X_{R 1}}(t)=\left(\frac{1}{2 \varepsilon}\left(\sqrt{-(\varepsilon-4) \varepsilon} \tan \left(\frac{1}{2} t \sqrt{-(\varepsilon-4) \varepsilon}+\tan ^{-1}\left(\frac{\left(2 x_{0}-1\right) \sqrt{\varepsilon}}{\sqrt{4-\varepsilon}}\right)\right)+\varepsilon\right),\right. \\
& \frac{1}{2 g^{\omega}}\left(\sqrt { 4 e ^ { \omega } g ^ { \omega } - ( f ^ { \omega } ) ^ { 2 } } \operatorname { t a n } \left(\frac{1}{2} t \sqrt{4 e^{\omega} g^{\omega}-\left(f^{\omega}\right)^{2}}\right.\right. \\
& \left.\left.\left.+\tan ^{-1}\left(\frac{f^{\omega}+2 g^{\omega} y_{0}}{\sqrt{4 e^{\omega} g^{\omega}-\left(f^{\omega}\right)^{2}}}\right)\right)-f^{\omega}\right)\right), \phi_{X_{R 2}}(t) \\
& =\left(\frac { 1 } { 2 ( g ^ { \omega } ) ^ { 2 } } \left(-\left(\eta_{1} g^{\omega}-f^{\omega} \eta_{2}\right)\left(2 \operatorname { l o g } \left(\operatorname { c o s } \left(\frac{1}{2} t \sqrt{4 e^{\omega} g^{\omega}-\left(f^{\omega}\right)^{2}}+\right.\right.\right.\right.\right. \\
& \left.\left.\left.\tan ^{-1}\left(\frac{f^{\omega}+2 g^{\omega} y_{0}}{\sqrt{4 e^{\omega} g^{\omega}-\left(f^{\omega}\right)^{2}}}\right)\right)\right)+\log \left(1-\frac{\left(f^{\omega}+2 g^{\omega} y_{0}\right)^{2}}{\left(f^{\omega}\right)^{2}-4 e^{\omega} g^{\omega}}\right)\right)+ \\
& \eta_{2} \sqrt{4 e^{\omega} g^{\omega}-\left(f^{\omega}\right)^{2}} \tan \left(\frac{1}{2} t \sqrt{4 e^{\omega} g^{\omega}-\left(f^{\omega}\right)^{2}}+\tan ^{-1}\left(\frac{f^{\omega}+2 g^{\omega} y_{0}}{\sqrt{4 e^{\omega} g^{\omega}-\left(f^{\omega}\right)^{2}}}\right)\right)- \\
& \left.g^{\omega}\left(2 \eta_{2}\left(e^{\omega} t+y_{0}\right)+f^{\omega} \eta_{1} t\right)+f^{\omega} \eta_{2}\left(f^{\omega} t-1\right)+2\left(g^{\omega}\right)^{2}\left(t+x_{0}\right)\right) \text {, } \\
& \frac{1}{2 g^{\omega}}\left(\sqrt { 4 e ^ { \omega } g ^ { \omega } - ( f ^ { \omega } ) ^ { 2 } } \operatorname { t a n } \left(\frac{1}{2} t \sqrt{4 e^{\omega} g^{\omega}-\left(f^{\omega}\right)^{2}}+\right.\right. \\
& \left.\left.\left.\tan ^{-1}\left(\frac{f^{\omega}+2 g^{\omega} y_{0}}{\sqrt{4 e^{\omega} g^{\omega}-\left(f^{\omega}\right)^{2}}}\right)\right)-f^{\omega}\right)\right)
\end{aligned}
$$


respectively.

$$
\begin{aligned}
\phi_{X_{R 3}}(t)= & \left(\frac{1}{\pi} \tan ^{-1}\left(\frac{(\varepsilon+1) \tanh \left(\pi t \sqrt{\varepsilon^{2}-1}+\tanh ^{-1}\left(\frac{(\varepsilon-1) \tan \left(\pi x_{0}\right)}{\sqrt{\varepsilon^{2}-1}}\right)\right)}{\sqrt{\varepsilon^{2}-1}}\right),\right. \\
& \frac{1}{2 g^{\omega}}\left(\sqrt { 4 e ^ { \omega } g ^ { \omega } - ( f ^ { \omega } ) ^ { 2 } } \operatorname { t a n } \left(\frac{1}{2} t \sqrt{4 e^{\omega} g^{\omega}-\left(f^{\omega}\right)^{2}}+\right.\right. \\
& \left.\left.\left.\tan ^{-1}\left(\frac{f^{\omega}+2 g^{\omega} y_{0}}{\sqrt{4 e^{\omega} g^{\omega}-\left(f^{\omega}\right)^{2}}}\right)\right)-f^{\omega}\right)\right),
\end{aligned}
$$

In the following we detail the proof for $X_{L L}$. In this case considering the flow $\phi_{X_{L-}}(t)=$ $\left(x_{1}(t), y_{1}(t)\right)$ starting at the point $p=\left(x_{0}, 0\right) \in \Sigma_{1}$, the smallest positive time $t_{1}(p)$ such that $\phi_{X_{L-}}\left(t_{1}(p)\right) \in \Sigma_{2}$ is

$$
t_{1}(p)=\frac{\log \left(\frac{c^{-}}{2 d^{-}}+1\right)}{c^{-}} .
$$

In this way we obtain the half first return map $P_{L}^{-}: \Sigma_{1} \rightarrow \Sigma_{2}$ given by $P_{1}^{-}\left(x_{0}, 0\right)=\phi_{X_{L-}}\left(t_{1}(p)\right)$ $=\left(x_{1}, 1 / 2\right)$.

Considering now the flow $\phi_{X_{L+}}(t)=\left(x_{2}(t), y_{2}(t)\right)$ and the initial condition $p_{1}=\left(x_{1}, 1 / 2\right)$ the smallest positive time such that $\phi_{X_{L+}}\left(t\left(p_{1}\right)\right) \in \Sigma_{2}$ is

$$
t_{2}\left(p_{1}\right)=\frac{\log \left(\frac{c^{+}}{c^{+}+2 d^{+}}+1\right)}{c^{+}},
$$

that provides the upper half first return map $P_{1}^{+}: \Sigma_{2} \rightarrow \Sigma_{1}$ given by $P_{L}^{+}\left(x_{1}, 1 / 2\right)=\phi_{X_{L+}}\left(t_{2}\left(p_{1}\right)\right.$ $=\left(x_{2}, 0\right)$. Note that a sufficient condition in order that $t_{1}(p)$ and $t_{2}\left(p_{1}\right)$ are the smallest positive times is that $X_{L L} \in\left[\Omega_{L^{+}}^{1} \cup \Omega_{L^{+}}^{2}\right] \cap\left[\Omega_{L^{-}}^{1} \cup \Omega_{L^{-}}^{2}\right]$.

Finally the first return map $P_{L L}: \Sigma_{1} \rightarrow \Sigma_{1}$ is given by the composition $P_{L L}\left(x_{0}\right)=\left(P_{L}^{+} \circ\right.$ $\left.P_{L}^{-}\right)\left(x_{0}\right)=x_{0}+\Delta_{L L}$.

Working in a similar way as in the computation of the first return map $P_{L L}$, we have obtained for the other first return maps their domains of definition and their expressions.

4.2. Proof of Theorem 2. Now we are able to perform the proof of Theorem 2. Let $P_{X}$ be the first return map for each PSVF $X$ consider in this paper. Now we define the displacement map

$$
d_{X}\left(x_{0}\right)=P_{X}\left(x_{0}\right)-x_{0}
$$

The limit cycles of $X$ are given by simple zeros of $d_{X}$. Lemma 5 provides the first return map for PSVF. Thus the proof of statements $(a),(b),(c),(d),(f),(i)$ and $(l)$ follows directly because in each one of these cases the first return map is given by $P_{X}\left(x_{0}\right)=x_{0}+\Delta_{X}$ where $\Delta_{X}$ is a real number given in function of coefficients of $X$. Therefore the iterates of $P_{X}$ are $P_{X}^{k}\left(x_{0}\right)=x_{0}+k \Delta_{X}$, or equivalently the $k$-iterate of the displacement map is $d_{X}^{k}\left(x_{0}\right)=k \Delta_{X}$, where $k$ is an integer number. Considering the equivalence relation (2) that defines the two dimensional torus, we have that $d_{X}^{k}\left(x_{0}\right)$ return to $x_{0}$ if and only if there exists an integer $k_{0}$ such that $k_{0} \Delta_{X} \in \mathbb{Z}$, or equivalently $\Delta_{X}$ is a rational number. Otherwise if $\Delta_{X}$ is not a rational number then the trajectory passing through $X_{0}$ never closes. In other words, $P_{X}^{k}$ is a rotation on the circle with irrational rotation number, so we conclude that all trajectories are dense in torus and the proof follows for these cases. 
Jaume, por favor, veja se o argumento usado acima esta bom.

In the following we detail the proofs of statements $(e)$ and $(h)$.

The first return map for $X_{R R 1+}$ is given in statement $(e)$ of Lemma 5 , so the displacement map in this case is

$$
d_{X R R 1+}\left(x_{0}\right)=\frac{1}{2}\left(\frac{\sqrt{4-\varepsilon} \tan \left(\xi_{1}-\tan ^{-1}\left(\xi_{2}+\frac{\left(1-2 x_{0}\right) \sqrt{\varepsilon}}{\sqrt{4-\varepsilon}}\right)\right)}{\sqrt{\varepsilon}}-2 x_{0}+1\right),
$$

where $\xi_{1}=\frac{\sqrt{(4-\varepsilon) \varepsilon}\left(\tan ^{-1}\left(\theta_{1}^{+}\right)-\sec ^{-1}\left(\theta_{3}^{+}\right)\right)}{\sqrt{4 e^{+} g^{+}-\left(f^{+}\right)^{2}}}$ and $\xi_{2}=\frac{4 \sqrt{\varepsilon}\left(\sec ^{-1}\left(\theta_{3}^{-}\right)-\tan ^{-1}\left(\theta_{2}^{+}\right)\right)}{\sqrt{4-\varepsilon} \sqrt{4 e^{-} g^{-}-\left(f^{-}\right)^{2}}}$ if $\varepsilon>0$. As $X_{R R 1+} \in\left[\Omega_{R^{-}}^{2} \cap \Omega_{R^{+}}^{3}\right]$ then $\xi_{1}>0$ and $\xi_{2}>0$. Solving directly the equation $d_{X R R 1+}\left(x_{0}\right)=0$ we obtain the values

$$
x_{0}^{ \pm}=\frac{ \pm \csc \left(\xi_{1}\right) \sqrt{(4-\varepsilon) \varepsilon \sin ^{2}\left(\xi_{1}\right)\left(4 \xi_{2} \cot \left(\xi_{1}\right)+\xi_{2}^{2}-4\right)}+\xi_{2} \sqrt{(4-\varepsilon) \varepsilon}+2 \varepsilon}{4 \varepsilon},
$$

recall that $\varepsilon>0$.

In fact, the radical $R_{R R 1+}=(4-\varepsilon) \varepsilon \sin ^{2}\left(\xi_{1}\right)\left(4 \xi_{2} \cot \left(\xi_{1}\right)+\xi_{2}^{2}-4\right)$ is given in function of $\varepsilon$ and can be written as

$$
R_{R R 1+}(\varepsilon)=\frac{16 \varepsilon^{2}\left(\left(\sec ^{-1}\left(\theta_{3}^{+}\right)-\tan ^{-1}\left(\theta_{1}^{+}\right)\right)^{2}\left(\frac{4 \sqrt{4 e^{+} g^{+}+\left(f^{+}\right)^{2}}\left(\tan ^{-1}\left(\theta_{2}^{-}\right)-\sec ^{-1}\left(\theta_{3}^{-}\right)\right)}{\sqrt{4 e^{-} g^{-}-\left(f^{-}\right)^{2}}\left(\tan ^{-1}\left(\theta_{1}^{+}\right)-\sec ^{-1}\left(\theta_{3}^{+}\right)\right)}-4\right)\right)}{\left(f^{+}\right)^{2}-4 e^{+} g^{+}}+\mathcal{O}\left(\varepsilon^{5 / 2}\right) .
$$

Since $X_{R R 1+} \in\left[\Omega_{R^{-}}^{2} \cap \Omega_{R^{+}}^{3}\right]$ we have that $R_{R R 1+}$ is positive. Therefore if $\varepsilon>0$ there exists two simple zeros of $d_{X R R 1+}$, or equivalently two limit cycles of $X_{R R 1+}$. In Example 6 we show a PSVF $X_{R R 1+}$ presenting exactly two limit cycles.

For the case $(h)$ the displacement map of $X_{L R 1-}$ is

$$
d_{X L R 1-}\left(x_{0}\right)=\frac{1}{2}\left(\xi_{3}-2 x_{0}+\frac{\tanh \left(\xi_{4}+\tanh ^{-1}\left(\left(2 x_{0}-1\right) \sqrt{\frac{\varepsilon}{\varepsilon-4}}\right)\right)}{\sqrt{\frac{\varepsilon}{\varepsilon-4}}}\right)
$$

where $\xi_{3}=\frac{2 \log \left(\frac{c^{+}}{c^{+}+2 d^{+}}+1\right)\left(b^{+} c^{+}-a^{+} d^{+}\right)+c^{+}\left(a^{+}+c^{+}\right)}{\left(c^{+}\right)^{2}}$ and $\xi_{4}=\frac{\sqrt{4-\varepsilon} \sqrt{-\varepsilon}\left(\tan ^{-1}\left(\theta_{2}^{-}\right)-\sec ^{-1}\left(\theta_{3}^{-}\right)\right)}{\sqrt{4 e^{-} g^{-}-\left(f^{-}\right)^{2}}}$ for $X_{L R 1-} \in\left[\Omega_{L^{+}}^{1} \cup \Omega_{L^{+}}^{2}\right] \cap \Omega_{R^{-}}^{2}$ and $\varepsilon<0$. Note that $\xi_{3}>0$. Solving the equation $d_{X L R 1-}\left(x_{0}\right)=0$ we obtain for $x_{0}$ the values

$$
x_{0}^{ \pm}= \pm \frac{\sqrt{\varepsilon\left(\frac{4\left(\xi_{3}-1\right) \varepsilon \operatorname{coth}\left(\xi_{4}\right)}{\sqrt{\frac{\varepsilon}{\varepsilon-4}}}+\left(\left(\xi_{3}-2\right) \xi_{3}+5\right) \varepsilon-16\right)}+\xi_{3} \varepsilon+\varepsilon}{4 \varepsilon} .
$$

These values of $x_{0}$ are real numbers because $\varepsilon<0, a^{+} c^{+}>0$ and $b^{+} c^{+}>a^{+} d^{+}$. Moreover, the radical $R_{L R 1-}=\varepsilon\left(\frac{4\left(\xi_{3}-1\right) \varepsilon \operatorname{coth}\left(\xi_{4}\right)}{\sqrt{\frac{\varepsilon}{\varepsilon-4}}}+\left(\left(\xi_{3}-2\right) \xi_{3}+5\right) \varepsilon-16\right)$ in terms of $\varepsilon$ can be written as

$$
\sqrt{-\varepsilon}\left(\sqrt{\frac{4 \sqrt{4 e^{-} g^{-}-\left(f^{-}\right)^{2}}\left(2 \log \left(\frac{c^{+}}{c^{+}+2 d^{+}}+1\right)\left(b^{+} c^{+}-a^{+} d^{+}\right)+a^{+} c^{+}\right)}{\left(c^{+}\right)^{2}\left(\tan ^{-1}\left(\theta_{2}^{-}\right)-\sec ^{-1}\left(\theta_{3}^{-}\right)\right)}+16}\right)+\mathcal{O}\left(\varepsilon^{3 / 2}\right) .
$$


So assuming that $X_{L R 1-} \in\left[\Omega_{L^{+}}^{1} \cup \Omega_{L^{+}}^{2}\right] \cap \Omega_{R^{-}}^{2}, \varepsilon<0, a^{+} c^{+}>0$ and $b^{+} c^{+}>a^{+} d^{+}$, then $R_{L R 1-}>0$. A vector field $X_{L R 1-}$ with exactly two limit cycle is presented in Example 7 .

For the case $(g)$, similarly to the previous cases, the displacement map for $X_{R R 3+}$ is

$$
d_{X R R 3+}\left(x_{0}\right)=-\frac{1}{\pi}\left(\tan ^{-1}\left(\frac{\sqrt{\varepsilon+1} \tan \left(\xi_{5}+\tan ^{-1}\left(\frac{(\varepsilon-1) \tan \left(\xi_{6}+\pi x_{0}\right)}{\sqrt{1-\varepsilon^{2}}}\right)\right)}{\sqrt{1-\varepsilon}}\right)\right)-x_{0},
$$

where $\xi_{5}=\frac{2 \pi \sqrt{1-\varepsilon^{2}}\left(\tan ^{-1}\left(\theta_{2}^{+}\right)-\tan ^{-1}\left(\theta_{1}^{+}\right)\right)}{\sqrt{4 e^{+} g^{+}-\left(f^{+}\right)^{2}}}$ and $\xi_{6}=\frac{2 \pi\left(\tan ^{-1}\left(\theta_{2}^{-}\right)-\sec ^{-1}\left(\theta_{3}^{-}\right)\right)}{\sqrt{4 e^{-} g^{-}-\left(f^{-}\right)^{2}}}$. As $X_{R R 3+} \in$ $\left[\Omega_{R^{-}}^{2} \cap \Omega_{R^{+}}^{1}\right]$ then $\xi_{5}<0$ and $\xi_{6}>0$. The solutions of equation $d_{X R R 3+}\left(x_{0}\right)=0$ are

$$
\begin{aligned}
& x_{0}^{1}=\frac{\cos ^{-1}\left(\frac{\sqrt{1-\varepsilon^{2}} \cot \left(\xi_{5}\right) \sin \left(\xi_{6}\right)-\cos \left(\xi_{6}\right)}{\varepsilon}\right)-\xi_{6}}{2 \pi}+k_{1}, \\
& x_{0}^{2}=\frac{-\cos ^{-1}\left(\frac{\sqrt{1-\varepsilon^{2}} \cot \left(\xi_{5}\right) \sin \left(\xi_{6}\right)-\cos \left(\xi_{6}\right)}{\varepsilon}\right)-\xi_{6}}{2 \pi}+k_{2},
\end{aligned}
$$

where $k_{1}, k_{2}$ are integer numbers. In the torus we obtain only two distinct points and the integers $k_{1}$ and $k_{2}$ are the smallest such that $x_{0}^{1}, x_{0}^{2} \in[0,1]$. In Example 8 we perform a PSVF with two limit cycles.

Remain to prove the statement $(j)$ when $X_{L R 3-} \in\left[\Omega_{L^{+}}^{1} \cup \Omega_{L^{+}}^{2}\right] \cap \Omega_{R^{-}}^{2}$. In this case the displacement map is

$$
d_{X L R 3-}\left(x_{0}\right)=\frac{1}{\pi}\left(\tan ^{-1}\left(\frac{\sqrt{\varepsilon+1} \tan \left(\xi_{7}-\tan ^{-1}\left(\frac{(\varepsilon-1) \tan \left(\pi x_{0}\right)}{\sqrt{1-\varepsilon^{2}}}\right)\right)}{\sqrt{1-\varepsilon}}\right)\right)+\xi_{8}-x_{0},
$$

where $\xi_{7}=\frac{2 \pi \sqrt{1-\varepsilon^{2}}\left(\tan ^{-1}\left(\theta_{2}^{-}\right)-\sec ^{-1}\left(\theta_{3}^{-}\right)\right)}{\sqrt{4 e^{-} g^{-}-\left(f^{-}\right)^{2}}}$ and $\xi_{8}=\frac{2 \log \left(\frac{c^{+}}{c^{+}+2 d^{+}}+1\right)\left(b^{+} c^{+}-a^{+} d^{+}\right)+a^{+} c^{+}}{2\left(c^{+}\right)^{2}}$. As $X_{L R 3-} \in$ $\left[\Omega_{L^{+}}^{1} \cup \Omega_{L^{+}}^{2}\right] \cap \Omega_{R^{-}}^{2}$ then $\xi_{7}>0$.

Considering the change of coordinates $z=\tan \left(\pi x_{0}\right)$ the map $d_{X L R 3-}$ can be written

$$
d_{X L R 3-}(z)=\frac{C_{1} z(\varepsilon+1)}{C_{1} \varepsilon+C_{1}-z \sqrt{1-\varepsilon^{2}}}+\frac{\varepsilon+1}{C_{1} \sqrt{1-\varepsilon^{2}}+z(\varepsilon-1)}-\frac{C_{2} z}{C_{2}+z}+\frac{1}{C_{2}+z},
$$

where we denote by $C_{1}=\cot \left(\xi_{7}\right)$ and $C_{2}=\cot \left(\xi_{8} \pi\right)$. Observe that $d_{X L R 3-}(z)$ is given as a linear combination of the functions $g_{0}(z)=1 /\left(C_{2}+z\right), g_{1}(z)=z /\left(C_{2}+z\right)$ and $g_{2}(z)=$ $1 /\left(C_{1} \sqrt{1-\varepsilon^{2}}+z(\varepsilon-1)\right)$. In fact, it is sufficient to prove that the function $g_{3}(z)=z /\left(C_{1} \varepsilon+\right.$ $\left.C_{1}-z \sqrt{1-\varepsilon^{2}}\right)$ is given as a linear combination of the functions $g_{0}, g_{1}$ and $g_{2}$. By a direct computation we obtain that the Wronskian $W_{3}\left(g_{0}, g_{1}, g_{2}, g_{3}\right)(z)$ is zero. Therefore the set of functions $\left\{g_{0}(z), g_{1}(z), g_{2}(z), g_{3}(z)\right\}$ is linearly dependent.

Besides than if we consider the ordered set of functions $\mathcal{F}=\left\{g_{0}(z), g_{1}(z), g_{2}(z)\right\}$, the Wronskians $W_{1}(z)$ and $W_{2}(z)$ are

$$
\begin{aligned}
& W_{1}(z)=\frac{1}{\left(C_{2}+z\right)^{2}}, \\
& W_{2}(z)=\frac{2(\varepsilon-1)\left(C_{2}(\varepsilon-1)-C_{1} \sqrt{1-\varepsilon^{2}}\right)}{\left(C_{2}+z\right)^{3}\left(C_{1} \sqrt{1-\varepsilon^{2}}+z(\varepsilon-1)\right)^{3}} .
\end{aligned}
$$


So $W_{1}(z) \neq 0$ and $W_{2}(z) \neq 0$, because $\cot \left(\xi_{8} \pi\right) \neq-\cot \left(\xi_{7}\right) \sqrt{\frac{1+\varepsilon}{1-\varepsilon}}$, i.e.

$$
\begin{aligned}
& \cot \left(\frac{\pi\left(2 \log \left(\frac{c^{+}}{c^{+}+2 d^{+}}+1\right)\left(b^{+} c^{+}-a^{+} d^{+}\right)+a^{+} c^{+}\right)}{2\left(c^{+}\right)^{2}}\right) \neq \\
& \cot \left(\frac{2 \pi \sqrt{1-\varepsilon^{2}}\left(\tan ^{-1}\left(\theta_{2}^{-}\right)-\sec ^{-1}\left(\theta_{3}^{-}\right)\right)}{\sqrt{4 e^{-} g^{-}-\left(f^{-}\right)^{2}}}\right)\left(\sqrt{\frac{1+\varepsilon}{1-\varepsilon}}\right) .
\end{aligned}
$$

Therefore, by Theorem B we obtain that the upper bound of zeros of any linear combination of functions in $\mathcal{F}$ is two and besides that there exists a linear combination of $\mathcal{F}$ presenting exactly two zeros.

In this way, as the displacement map $d_{X L R 3-}$ is given as a specific linear combination of functions of $\mathcal{F}$ we guarantee that the upper bound of zeros of $d_{X L R 3-}$ is two (as a function of $\left.z=\tan \left(\pi x_{0}\right)\right)$. But if $z_{1}, z_{2}$ are the zeros of $d_{X L R 3-}$ then there exists $x_{0}^{1}+k_{1}$ and $x_{0}^{2}+k_{2}$ real numbers where $k_{1}, k_{2}$ are integer numbers such that $\tan \left(\pi\left(x_{0}^{1}+k_{1}\right)\right)=z_{1}$ and $\tan \left(\pi\left(x_{0}^{2}+k_{2}\right)\right)=$ $z_{2}$. We choose the integers $k_{1}$ and $k_{2}$ such that $x_{0}^{1}, x_{0}^{2} \in[0,1]$. Despite the displacement map $d_{X L R 3-}$ is a specific linear combination of $g_{0}, g_{1}$ and $g_{2}$ in Example 9 we are able to present values of the parameters such that $X_{L R 3-}$ presents exactly two limit cycles.

4.3. Proof of Theorem 3. Recall that $X_{C k}(x, y)=\left(X_{C}(x, y), X_{k}(x, y)\right.$ is defined on the torus, with $X_{C}(x, y)=X_{C}^{+}(x, y)=\left(b^{+}, d^{+}\right)$defined on $\Sigma^{+}$and $X_{k}(x, y)=(\alpha, \beta \cos (2 k \pi x)$ is defined on $\Sigma^{-}$.

It is straightforward to obtain the expressions of the flows $\phi_{X_{k}}(t)$ and $\phi_{X_{C}}(t)$ of $X_{k}$ and $X_{C}^{+}$ respectively, passing through the point $p=\left(x_{0}, y_{0}\right)$ when $t=0$, namely

$$
\begin{aligned}
& \phi_{X_{k}}(t)=\left(\alpha t+x_{0}, y_{0}-\frac{\beta \sin \left(2 \pi k x_{0}\right)}{2 k \pi \alpha} \frac{\beta \sin \left(2 \pi \alpha k t+2 \pi k x_{0}\right)}{2 k \pi \alpha}\right), \\
& \phi_{X_{C}^{+}}(t)=\left(b^{+} t+x_{0}, d^{+} t+y_{0}\right) .
\end{aligned}
$$

The fly maps $P_{X_{k}}:\{(x, y) \in Q ; y=0\} \rightarrow\{(x, y) \in Q ; y=1 / 2\}$ of $\phi_{X_{k}}$ and $P_{X_{C}}:\{(x, y) \in$ $Q ; y=1 / 2\} \rightarrow\{(x, y) \in Q ; y=1\}$ of $\phi_{X_{C}^{+}}$are given by

$$
\begin{aligned}
& P_{X_{k}}\left(x_{0}, 0\right)=\left(\frac{\arcsin \left(\frac{k \pi \alpha+\beta \sin \left(2 \pi k x_{0}\right)}{\beta}\right)}{2 k \pi}, \frac{1}{2}\right), \\
& P_{X_{C}^{+}}\left(x_{0}, 1 / 2\right)=\left(\frac{b^{+}+2 d^{+} x_{0}}{2 b}, 1\right),
\end{aligned}
$$

where $m \in\{0, \ldots, k\}$ is such that $x_{0} \in[m / k,(m+1) / k]$.

Thus the Poincaré map $P_{X_{C k}}:\{(x, y) \in Q ; y=0\} \rightarrow\{(x, y) \in Q ; y=1\}$ is given by $P_{X_{C k}}\left(x_{0}, 0\right)=\left(P_{X_{C}} \circ P_{X_{k}}\right)\left(x_{0}, 0\right)=\left(P_{1}\left(x_{0}\right), 1\right)$, with

$$
P_{1}\left(x_{0}\right)=\frac{1}{2 k \pi} \arcsin \left(\sin \left(2 k \pi x_{0}\right)+k \alpha \pi / \beta\right)+\frac{b^{+}}{2 d^{+}}+\frac{m}{k} .
$$

So to find limit cycles we have to find the simple zeros of the displacement map

$$
d_{X_{L k}}\left(x_{0}\right)=P_{1}\left(x_{0}\right)-x_{0},
$$


for $x_{0} \in[0,1]$. Now we show that for every $m=0, \ldots, k-1$, there are at most one solution for (7) with $x_{0} \in(m / k,(m+1) / k)$, thus there is at most $k$ limit cycles for $X_{L k}$.

Now we study the solutions of

$$
d^{+} \arcsin \left(\frac{k \pi \alpha+\beta \sin \left(2 \pi k x_{0}\right)}{\beta}\right)=2 x_{0} d^{+} k \pi-b^{+} k \pi-2 m d^{+} \pi
$$

for $x \in[0,1]$, where $m \in \mathbb{Z}$ and $x \in(m / k,(m+1) / k)$.

Fix $m=0$ and $k=1$ without loss of generality (we can always restrict ourselves to $x \in$ $[0,1 / k])$.

Before we conclude the analysis we discuss the tangency points of $X_{C k}$. For $k=1$, the tangency points are $(1 / 4,0),(3 / 4,0),(1 / 4,1 / 2)$ and $(3 / 4,1 / 2)$. According to the signs of $b^{+}$, $d^{+}, \alpha$, and $\beta$, the segments between these points vary between (stable or unstable) sliding segments and crossing segments.

Suppose $b^{+} \alpha<0$ and $d^{+} \beta<0$. Then the segments $[1 / 4,3 / 4] \times\{0\}$ and $[1 / 4,3 / 4] \times\{1 / 2\}$ are crossing regions. Solutions passing outside these segments cannot be limit cycles.

Note that the function $g\left(x_{0}\right)=d^{+} \arcsin \left(\frac{k \pi \alpha+\beta \sin \left(2 \pi k x_{0}\right)}{\beta}\right)$ has two critical points, $1 / 4$ and $3 / 4$, so it is monotone on $(1 / 4,3 / 4)$. Therefore the straight line $2 x_{0} d^{+} k \pi-b^{+} k \pi-2 m d^{+} \pi$ meets the graph of $g$ at most in one point. Thus there is at most 1 limit cycles for $X_{C k}$ with $k=1$. It is easy to see that there are at most $k$ limit cycles for $X_{C k}$. In Example 10 we provided values of the coefficients $\alpha, \beta, b^{+}$and $d^{+}$for which the PSVF $X_{C k}$ presents one limit cycle for $k=1$.

\section{Final Remarks and SOME EXAmples}

In the present section we exhibit explicit values for the parameters of the PSVFs $X_{R R 1+}$, $X_{R R 3+}, X_{L R 1-}$ and $X_{L R 3-}$ for which they realize their upper bound on the maximum number of limit cycles.

Example 6. If $e^{+}=0.466532, f^{+}=0.1, g^{+}=0.541227, e^{-}=-0.35481, f^{-}=0.4, g^{-}=$ -0.817339 and $\varepsilon=0.02$, then the displacement map associated to $X_{R R 1+}(x, y)$ given in (4) is

$$
d_{X R R 1+}\left(x_{0}\right)=7.05337 \tan \left(0.217-\tan ^{-1}\left(0.290888-0.141776 x_{0}\right)\right)-x_{0}+0.5 .
$$

Solving the equation $d_{X R R 1+}\left(x_{0}\right)=0$ we obtain the points $x_{0}^{1}=0.571897$ and $x_{0}^{2}=1.97984=$ $1+0.97984$ which represents the points in the torus: $y_{0}^{1}=0.571897$ and $y_{0}^{2}=0.97984$. In other words, this means that the trajectory passing through $y_{0}^{2}$ rotates one time before return to $y_{0}^{2}$.

Example 7. If $a^{+}=-4.47442, b^{+}=0, c^{+}=1, d^{+}=1, e^{-}=0.0355785, f^{-}=\sqrt{3}, g^{-}=$ 28.1069 and $\varepsilon=-0.08$, then the displacement map associated to $X_{L R 11}(x, y)$ given in (4) is

$$
d_{X L R 1-}\left(x_{0}\right)=-x_{0}+3.57071 \tanh \left(\tanh ^{-1}\left(0.140028\left(2 x_{0}-1\right)\right)+0.28\right)-0.45,
$$

and its solutions of $d_{X L R 1-}\left(x_{0}\right)=0$ in the torus are $x_{0}^{1}=0.286257$ and $x_{0}^{2}=0.763743$.

Example 8. If $e^{+}=0.584555, f^{+}=0.130158, g^{+}=0.434921, e^{-}=0.670355, f^{-}=\sqrt{3}$, $g^{-}=1.49175$ and $\varepsilon=0.06$, then the displacement map associated to $X_{R R 3+}(x, y)$ given in (4) is

$$
d_{X R R 3+}\left(x_{0}\right)=\frac{\tan ^{-1}\left(1.06191 \tan \left(\tan ^{-1}\left(0.941697 \tan \left(\pi x_{0}+1.4\right)\right)+1.7\right)\right)}{\pi}-x_{0},
$$

and the solutions of $d_{X R R 3+}\left(x_{0}\right)=0$ in the torus are $x_{0}^{1}=0.15119$ and $x_{0}^{2}=0.403176$. 
Example 9. If $a^{+}=1.21371, b^{+}=1, c^{+}=1, d^{+}=0, e^{-}=0.250971, f^{-}=\sqrt{3}, g^{-}=3.98453$ and $\varepsilon=0.04$, then the displacement map associated to $X_{L R 3-}(x, y)$ given in (4) is

$$
d_{X L R 3-}\left(x_{0}\right)=-x_{0}+0.31831 \tan ^{-1}\left(1.04083 \tan \left(\tan ^{-1}\left(0.960769 \tan \left(\pi x_{0}\right)\right)+2.2\right)\right)+1.3,
$$

and the solutions of $d_{X L R 3-}\left(x_{0}\right)=0$ in the torus are $x_{0}^{1}=0.397519$ and $x_{0}^{2}=0.902481$, see Figure 1.



Figure 1. The two limit cycles presented in Example 9.

Example 10. Finally we provide an example with exactly $k$ limit cycles for $X_{C k}$ (see Theorem 3) for $k=1$. Given the vector field $X_{k}(x, y)=(\alpha, \beta \cos (2 k \pi x)$ with $\alpha, \beta>0$ and $k>0$ an integer, we will construct a vector field $X_{C}(x, y)=\left(b^{+}, d^{+}\right)$with a limit cycle. Note that for every $m=0, \ldots, k-1$ we have

$$
P_{X_{k}}\left(\frac{1}{8 k}+\frac{m}{k}, \frac{1}{2}\right)=\left(\frac{1}{2 k \pi} \arcsin \left(\frac{k \pi \alpha}{\beta}+\frac{\sqrt{2}}{2}\right)+\frac{m}{k}, \frac{1}{2}\right) .
$$

So the first restriction is $-1<\frac{k \pi \alpha}{\beta}+\frac{\sqrt{2}}{2}<1$. Now we fix $m=0$ and prove that we have at least limit cycles for $x \in[0,1 / k]$.

$$
\text { Let } \Delta_{C k}=\frac{1}{8 k}-\frac{1}{2 k \pi} \arcsin \left(\frac{2 k \pi \alpha+\beta \sqrt{2}}{2 \beta}\right) \text { and consider } X_{C}(x, y)=\left(\Delta_{C k}, 1 / 2\right), \text { i.e. } b^{+}=
$$

$\Delta_{C k}$ and $d^{+}=1 / 2$. By construction, $P_{X_{C k}}(1 / 8 k, 0)=(1 / 8 k, 1)$, so we have a fixed point of the Poincaré map of $X_{C k}$, and consequently a limit cycle. The derivative of the Poincaré map on this fixed point is

$$
P_{1}^{\prime}(1 / 8 k)=\left(\sqrt{\frac{\beta^{2}-2 \sqrt{2} \pi \alpha \beta k-2 \pi^{2} \alpha^{2} k^{2}}{\beta^{2}}}\right)^{-1}
$$

that is not zero under generic conditions. So this is an isolated fixed point, providing a limit cycle, see Figure 2. Thus we have exactly $k=1$ limit cycles. 


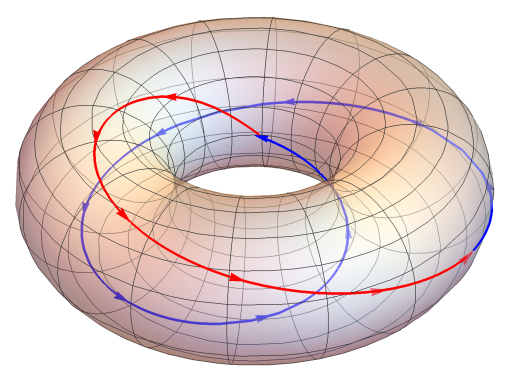

Figure 2. The only limit cycle for $X_{C k}$ with $k=1$ in Example 10 .

\section{ACKNOWLEDGEMENTS}

The first author is partially supported by the MINECO grants MTM2016-77278-P and MTM2013-40998-P, an AGAUR grant number 2014SGR-568, the grants FP7-PEOPLE-2012IRSES 318999, and a CAPES grant number 88881.030454/ 2013-01 from the program CSFPVE. D. J. Tonon is supported by grant\#2012/10 267000 803, Goiás Research Foundation (FAPEG), PROCAD/CAPES grant 88881.0 68462/2014-01 and by CNPq-Brazil. R. M. Martins is supported by FAPESP-Brazil project 2015/06903-8. The authors would like to thank Matheus Manzatto for the help with the figures.

\section{REFERENCES}

[1] R. Adler, B. Kitchens, and C. Tresser. Dynamics of non-ergodic piecewise affine maps of the torus. Ergodic Theory Dynam. Systems, 21(4):959-999, 2001.

[2] P. Ashwin, X. Fu, and C. Lin. On planar piecewise and two-torus parabolic maps. Internat. J. Bifur. Chaos Appl. Sci. Engrg., 19(7):2383-2390, 2009.

[3] N. I. Chernov. Ergodic and statistical properties of piecewise linear hyperbolic automorphisms of the 2-torus. J. Statist. Phys., 69(1-2):111-134, 1992.

[4] M. di Bernardo, C. J. Budd, A. R. Champneys, and P. Kowalczyk. Piecewise-smooth dynamical systems, volume 163 of Applied Mathematical Sciences. Springer-Verlag London, Ltd., London, 2008. Theory and applications.

[5] A. F. Filippov. Differential equations with discontinuous righthand sides, volume 18 of Mathematics and its Applications (Soviet Series). Kluwer Academic Publishers Group, Dordrecht, 1988. Translated from the Russian.

[6] S. Karlin and W. J. Studden. Tchebycheff systems: With applications in analysis and statistics. Pure and Applied Mathematics, Vol. XV. Interscience Publishers John Wiley \& Sons, New York-London-Sydney, 1966.

[7] O. Makarenkov and J. S. W. Lamb. Dynamics and bifurcations of nonsmooth systems: a survey. Phys. D, 241(22):1826-1844, 2012.

[8] D. D. Novaes and J. A. Torregrosa. On extended chebyshev systems with positive accuracy. to appear in J. Math. Anal. Appl.

[9] Y. V. Orlov. Discontinuous systems. Communications and Control Engineering Series. Springer-Verlag London, Ltd., London, 2009. Lyapunov analysis and robust synthesis under uncertainty conditions.

[10] H. Poincaré. Mémoire sur les courbes d éfinies par une équation differentielle i, ii,. J. Math. Pures Appl., 7,8:375-422, 251-296, 1881, 1882.

[11] H. Poincaré. Sur les courbes définies par les équation differentielles iii, iv. J. Math. Pures Appl., 1,2:167-244, $155-217,1885,1886$. 
[12] D.J.W. Simpson. Bifurcations in Piecewise-Smooth Continuous Systems, volume 69 of World Scientific Series on Nonlinear Science A. World Scientific, Singapore, 2010.

[13] M. A. Teixeira. Perturbation theory for non-smooth systems. In Mathematics of complexity and dynamical systems. Vols. 1-3, pages 1325-1336. Springer, New York, 2012.

[14] V. I. Tkachenko. On the existence of a piecewise-smooth invariant torus of an impulse system. In Methods for investigating differential and functional-differential equations (Russian), pages 91-96. Akad. Nauk Ukrain. SSR, Inst. Mat., Kiev, 1990.

[15] D. J. Tonon and R. M. Martins. Chaos in piecewise smooth vector fields on two dimensional torus and sphere. https://arxiv.org/abs/1601.05670.

1 Departament de Matemàtiques, Universitat Autoǹoma de Barcelona, 08193 Bellaterra, Barcelona, SPAIN

E-mail address: jllibre@mat.uab.cat

2 IMECC-UNiCAMP, CEP 13083-859, CAmpinas, SÃo Paulo, Brazil

E-mail address: rmiranda@ime.unicamp.br

3 Institute of Mathematics and Statistics of Federal University of Goiás, Avenida Esperança s/n, Campus Samambaia, CEP 74690-900, Goin̂nia, Goiás, Brazil.

E-mail address: djtonon@ufg.br 